\title{
Psychological Science
}

Reduced Sensitivity to Visual Looming Inflates the Risk Posed by Speeding Vehicles When Children Try to Cross the Road

John P. Wann, Damian R. Poulter and Catherine Purcell

Psychological Science 2011 22: 429 originally published online 9 March 2011

DOI: $10.1177 / 0956797611400917$

The online version of this article can be found at:

http://pss.sagepub.com/content/22/4/429

Published by:

(\$)SAGE

http://www.sagepublications.com

On behalf of:

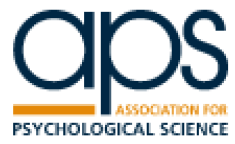

Association for Psychological Science

Additional services and information for Psychological Science can be found at:

Open Access: Immediate free access via SAGE Choice

Email Alerts: http://pss.sagepub.com/cgi/alerts

Subscriptions: http://pss.sagepub.com/subscriptions

Reprints: http://www.sagepub.com/journalsReprints.nav

Permissions: http://www.sagepub.com/journalsPermissions.nav

>> Version of Record - Apr 12, 2011

OnlineFirst Version of Record - Mar 17, 2011

OnlineFirst Version of Record - Mar 9, 2011

What is This? 


\title{
Reduced Sensitivity to Visual Looming Inflates the Risk Posed by Speeding Vehicles When Children Try to Cross the Road
}

Psychological Science 22(4) 429-434 (c) The Author(s) 2011 Reprints and permission: sagepub.com/journalsPermissions.nav DOI: $10.1177 / 095679761 \mid 400917$ http://pss.sagepub.com @SAGE

\author{
John P. Wann, Damian R. Poulter, and Catherine Purcell \\ Royal Holloway, University of London
}

\begin{abstract}
Almost all locomotor animals respond to visual looming or to discrete changes in optical size. The need to detect and process looming remains critically important for humans in everyday life. Road traffic statistics confirm that children up to 15 years old are overrepresented in pedestrian casualties. We demonstrate that, for a given pedestrian crossing time, vehicles traveling faster loom less than slower vehicles, which creates a dangerous illusion in which faster vehicles may be perceived as not approaching. Our results from perceptual tests of looming thresholds show strong developmental trends in sensitivity, such that children may not be able to detect vehicles approaching at speeds in excess of $20 \mathrm{mph}$. This creates a risk of injudicious road crossing in urban settings when traffic speeds are higher than $20 \mathrm{mph}$. The risk is exacerbated because vehicles moving faster than this speed are more likely to result in pedestrian fatalities.
\end{abstract}

\section{Keywords}

vision, detection, looming, children, road, safety, time to contact, tau, perception, development

Received 9/17/10; Revision accepted II/5/10

The ability to detect and avoid looming objects is critical to survival. Almost all locomotor animals are sensitive to visual looming or changes in optical size (Field \& Wann, 2005; Peron \& Gabbiani, 2009; Sun \& Frost, 1998). Sensitivity to optical expansion is critical for selection of an appropriate response in order to avoid a collision (e.g., when crossing the road). Failure to detect and process looming information accurately can have serious consequences. Globally, pedestrian accidents are the third leading cause of death for 5- to 9-year-olds, and children's visual limitations in gauging speed and distance are cited as a key deficit contributing to such accidents (Toroyan $\&$ Peden, 2007). In the United Kingdom alone, there are more than 6,500 pedestrian casualties per annum, and $30 \%$ of the individuals killed are children ages 0 to 15 years (Department for Transport, 2010).

At the roadside, most everyday distance cues provide only relative estimates or are prone to considerable bias that varies with the terrain. For example, a pedestrian could use vertical elevation (height in the scene) to gauge the distance of an approaching car. But if the car is traveling at $30 \mathrm{mph}$, and $5 \mathrm{~s}$ is needed to complete a safe crossing, then just a $1^{\circ}$ decrease in the slope of the road can make the car's height in the scene equivalent to that of a car 4 times further away. In addition, binocular cues to the distance of a car that is traveling at
$30 \mathrm{mph}$ and is $5 \mathrm{~s}$ away are negligible (Tresilian, MonWilliams, \& Kelly, 1999).

Optical size and optical looming provide available and reliable indications of distance and relative speed. The time to passage (TTP) of an approaching object (i.e., the time it takes the object to reach the observer) can also be determined from optical size and looming. Considering these variables over time, $t$, the TTP for a vehicle approaching the observer at constant speed is the ratio of its distance, $z(t)$, and velocity, $v(t)$, and this ratio in turn can be perceptually detected by the ratio of optical size, $\theta(t)$, and the rate of looming, $\dot{\theta}(t)$ :

$$
T T P=\frac{z(t)}{v(t)}=\frac{\theta(t)}{\dot{\theta}(t)}
$$

The ratio in Equation 1 has been called tau (Lee, 1976), and considerable research has been conducted on adults' and skilled performers' use of tau in estimating TTP (e.g., McLeod \& Ross, 1983; Schiff \& Detwiler, 1979). However, there has

\section{Corresponding Author:}

John P. Wann, Department of Psychology, Royal Holloway, University of London, Egham, Surrey, TW20 0EX, United Kingdom

E-mail: j.p.wann@rhul.ac.uk 
been less consideration of perceptual thresholds for detection of looming (see Regan \& Beverley, 1979) and no investigation of children's ability to detect looming. Determining detection thresholds for looming is crucial, as estimates of speed and TTP can be reliable only if the rate of looming is above the perceptual threshold of the observer. If $\dot{\theta}(t)$ is below the observer's perceptual threshold, this estimate of TTP goes to infinity (i.e., the object would appear to be motionless).

A further problem, however, arises from optical geometry. The instantaneous value of $\dot{\theta}(t)$ is dictated by the size of the vehicle profile $(S)$, which could be its width or height, and the vehicle's speed and distance (small-angles approximation):

$$
\dot{\theta}(t)=\frac{S \times v(t)}{z^{2}(t)}
$$

From Equation 2, one can see that faster vehicles loom at a faster rate than slower vehicles if they are at the same distance from the observer. But if the observer needs a specific duration of TTP to cross the road (time to cross, $t_{\mathrm{c}}$ ), then substituting $z^{2}=\left(v \times t_{\mathrm{c}}\right)^{2}$ into Equation 2 gives

$$
\dot{\theta}(t)=\frac{S}{t_{\mathrm{c}}^{2} \times v(t)}
$$

This demonstrates an important change from Equation 2 because speed has moved from being in the numerator to being in the divisor. Thus, for a set crossing duration and vehicle profile, any increase in approach speed will actually lower the rate of looming presented to the observer. This sounds counterintuitive, but a faster vehicle must be much farther away to allow an equivalent crossing time, and as a consequence will be very small optically and also have a lower looming rate. The consequence of Equation 3 is that if the approach speed of a vehicle is higher, it is more likely to be seen as small and static in the scene and may not pop out. Equation 3 can also be reversed to show that if one knows an observer's perceptual threshold for looming, $\dot{\theta}_{\text {th }}$, then one can predict the maximum vehicle speed, $v_{\max }$, that would allow the observer to reliably gauge whether a suitable crossing time is available:

$$
v_{\max }=\frac{S}{t_{\mathrm{c}}^{2} \times \dot{\theta}_{\mathrm{th}}}
$$

The adult threshold for looming detection in driving scenes has previously been estimated to be approximately $0.08 \%$ s to 0.11\% (Cavallo \& Laurent, 1988; Hoffmann, 1994; Lee, 1976), although it has been demonstrated that, under strict psychophysical conditions, simple-edge motion can be detected at approximately $0.02 \%$ s (Regan \& Beverley, 1979). Developmental differences in accuracy of TTP estimation coincide with casualty statistics demonstrating younger children's increased vulnerability at the roadside (Hoffmann, Payne, \& Prescott, 1980). Little information, however, exists for children's perceptual ability to detect looming stimuli, which provides the basis for judgments of TTP. Estimates of children's sensitivity to looming have been derived post hoc from TTP data (Hoffmann, 1994), but no studies have directly investigated children's detection of looming. We devised a task that systematically measured children's looming-detection threshold under different presentation conditions, and we then converted threshold values to realworld speeds in order to draw conclusions about the limits of children's ability to evaluate vehicles' approach speeds.

\section{Method \\ Participants}

A total of 111 schoolchildren and 27 adults were recruited for the study. Participants who had high false-positive rates (see the Procedure and Stimuli section) or who failed to complete the task were excluded, so the number of participants in some conditions was less than the original sample size (see Supplementary Documentation in the Supplemental Material available online for sample demographics). All participants had normal or corrected-to-normal vision and were naive to the purposes of the study. Parental informed consent was obtained for all children in advance of the study, and each child provided verbal assent immediately prior to the start of the experiment. The study was approved by the ethics panel of the Department of Psychology, Royal Holloway, University of London.

\section{Procedure and stimuli}

We presented a perspective-correct visual simulation of approaching vehicles to adults and children (three developmental groups: 6-7 years, $8-9$ years, and $10-11$ years) under four conditions: In two conditions, the stimuli were presented in the fovea (central vision), and in the other two, extrafoveal stimuli tested detection just outside of central vision. Within each condition, there were both probe trials and null trials. In probe trials, the vehicle image changed in size and speed; these trials simulated approach at various rates of looming from $5 \mathrm{~s}$ away. In null trials, the car image remained static at the same optic size as the initial image for the equivalent probe trial; null trials were incorporated in order to determine the reliability of observers' responses. For each trial, participants were asked to verbally indicate whether they thought the car image expanded or stayed the same size. There was no time pressure on their response.

The computer-generated stimuli were scripted using Python and Vizard (Development Edition; WorldViz, Santa Barbara, $\mathrm{CA}$ ) and displayed at $60 \mathrm{~Hz}$. Photo-realistic stimuli of a car, approaching at different speeds on different trials, were presented against a road-scene background. In the simpler foveal condition (isotropic expansion), we presented a brief $(0.2 \mathrm{~s})$ simulation of a vehicle that moved directly forward (probe trial) or remained stationary (null trial; see Fig. 1 for a sample frame). This display represented the final $0.2 \mathrm{~s}$ that a pedestrian would see before making a crossing judgment, 


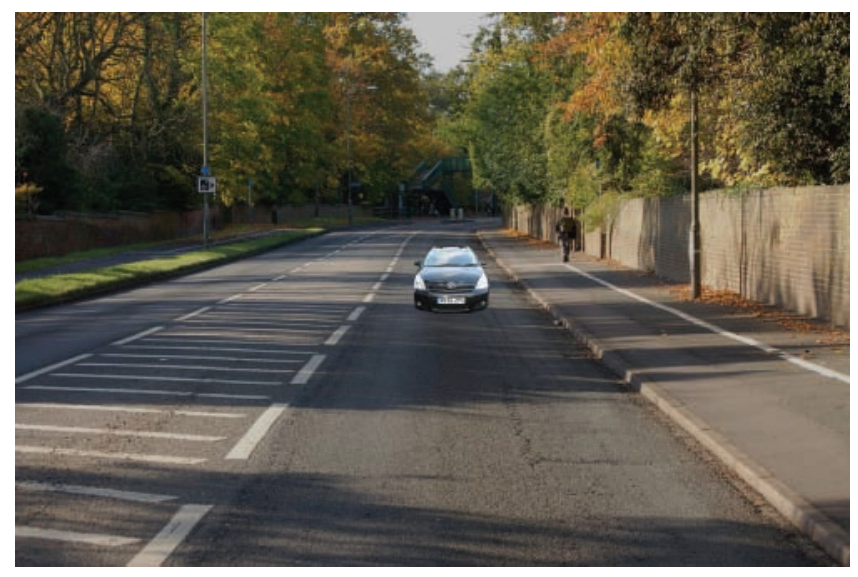

Fig. I. Example frame from a stimulus used to test thresholds for detecting looming in foveal vision. Each stimulus consisted of a $0.2-\mathrm{s}$ presentation of a photo-realistic image of a car against a contextual road-scene background. For the extrafoveal conditions, the car image was presented at $4.25^{\circ}$ eccentricity from the center of the screen.

while allowing for a $t_{\mathrm{c}}$ of $5 \mathrm{~s}$. In a natural setting, any sampling of the scene prior to this time would have had a lower rate of looming than that presented. In the simpler extrafoveal condition (isotropic expansion), the same stimuli were presented extrafoveally by positioning the car image $4.25^{\circ}$ from the central fixation point in one of four quadrants. Extrafoveal stimuli would occur in natural scenes if pedestrians did not fixate directly on a vehicle when visually scanning a cluttered street environment. For foveal stimuli, participants viewed the screen monocularly from a distance of $4 \mathrm{~m}$ so that the number of pixels per degree would be maximized, whereas for extrafoveal stimuli, they viewed the screen monocularly from $2 \mathrm{~m}$ to allow for the angular displacement. Images were automatically rescaled according to the viewing distance in order to ensure that visual angles were equivalent to those that would be experienced at the roadside.

A simulated $t_{\mathrm{c}}$ of $5 \mathrm{~s}$ was employed, as this was the length of time we estimated the children in our study would need to cross the width of a two-lane road safely without having to run (4.5-4.9 s). Participants' walking time was measured by asking them to walk a distance of $4.5 \mathrm{~m}$ in the laboratory. Mean walking time over three trials was multiplied by a factor of 1.2 in order to obtain an estimate of walking time required to cross a 5.4- $\mathrm{m}$ road width, which was the average of urban road widths measured in the areas where the road-scene backgrounds were photographed.

The first two conditions simulated direct approach of a vehicle but did not directly test the detection of looming, as observers could detect the movement of any edge or set of pixels. Therefore, we tested participants with two more conditions using a second set of stimuli that underwent the same isotropic expansion as the stimuli in the first set except that the image of the vehicle also underwent $1^{\circ}$ of lateral displacement, randomly oriented toward one of the four quadrants. These stimuli required observers to detect looming (isotropic expansion) isolated from simple edge motion; this additional lateral edge motion would result if the observer moved his or her head while trying to detect approaching vehicles. Thus, with this second set of stimuli, we tested participants' ability to detect expansion with lateral displacement in both foveal and extrafoveal conditions.

We estimated detection thresholds using a parameter estimation by sequential testing procedure (Best-PEST; Lieberman \& Pentland, 1982). Incremental changes in looming rate were simulated by first using Equation 3 to set a speed (meters/second) that would produce a specific rate of optical expansion (radians/second) while affording a TTP equal to $t_{\mathrm{c}}$ (set to $5 \mathrm{~s}$ ), and then setting approach distance by multiplying the speed by $t_{\mathrm{c}}$. The PEST staircases were stopped after the 10th reversal, and threshold was calculated as the average of the last 5 reversals. Trials with false-positive rates (i.e., frequency of incorrectly reporting movement in null trials) in excess of $33 \%$ for a single staircase procedure were deemed unreliable, and data from these trials were excluded from the final analysis. Further details on creation and presentation of the stimuli are available in the Method section of the Supplementary Documentation in the Supplemental Materials.

The effect of age group on detection threshold was analyzed using one-way ANOVA with age group (6-7 years, 8-9 years, 10-11 years, adults) as a between-subjects factor. Post hoc Tukey HSD tests were used for group comparisons.

\section{Results \\ Detection of isotropic expansion}

An ANOVA revealed a significant effect of age group on detection of looming in foveal vision, $F(3,126)=24.554, p<$ $.001, \eta_{p}{ }^{2}=.369$, and extrafoveal vision, $F(3,118)=20.129$, $p<.001, \eta_{p}{ }^{2}=.339$ (see Table 1 for summary statistics for all four conditions). Post hoc Tukey HSD analysis revealed that there was no significant difference in detection threshold for isotropic expansion in foveal vision between 6- to 7-year-olds and 8- to 9-year-olds. Children ages 10 to 11 years had a significantly lower (better) threshold than those ages 6 to 7 years, but had a threshold similar to that of children ages 8 to 9 years. Adults had a significantly lower (better) detection threshold than each of the three groups of children (see Table 2 for effect sizes and significance levels for all four conditions).

For isotropic expansion in extrafoveal vision, there was no difference in detection threshold between children ages 6 to 7 years and children ages 8 to 9 years, but both of these age groups had significantly higher (poorer) thresholds than children ages 10 to 11 years. Once again, adults had a significantly better threshold than each of the three groups of children. It is worth noting that thresholds for detecting looming stimuli presented in extrafoveal vision were higher (poorer) than thresholds in foveal vision even though our stimuli were presented only just outside the foveal field of vision. This demonstrates 
Table I. Descriptive Statistics for Untransformed Detection Thresholds for Each Condition and Age Group

\begin{tabular}{|c|c|c|}
\hline Condition and age group & $\begin{array}{c}M \\
\text { (degrees/second) }\end{array}$ & $95 \% \mathrm{Cl}$ \\
\hline \multicolumn{3}{|l|}{ Isotropic expansion: foveal } \\
\hline 6-7 years & $0.165(0.070)$ & $0.137-0.192$ \\
\hline $8-9$ years & $0.161(0.126)$ & $0.135-0.187$ \\
\hline$|0-1|$ years & $0.105(0.055)$ & $0.076-0.135$ \\
\hline Adult & $0.063(0.015)$ & $0.031-0.094$ \\
\hline \multicolumn{3}{|l|}{$\begin{array}{l}\text { Isotropic expansion: } \\
\text { extrafoveal }\end{array}$} \\
\hline 6-7 years & $0.332(0.103)$ & $0.296-0.369$ \\
\hline $8-9$ years & $0.333(0.141)$ & $0.299-0.366$ \\
\hline $10-1 \mid$ years & $0.239(0.072)$ & $0.20 I-0.277$ \\
\hline Adult & $0.189(0.052)$ & $0.148-0.230$ \\
\hline \multicolumn{3}{|l|}{$\begin{array}{l}\text { Expansion with lateral } \\
\text { displacement: foveal }\end{array}$} \\
\hline 6-7 years & $0.357(0.152)$ & $0.291-0.423$ \\
\hline $8-9$ years & $0.335(0.149)$ & $0.273-0.397$ \\
\hline$|0-1|$ years & $0.357(0.170)$ & $0.295-0.420$ \\
\hline Adult & $0.218(0.180)$ & $0.153-0.282$ \\
\hline \multicolumn{3}{|l|}{$\begin{array}{l}\text { Expansion with lateral } \\
\text { displacement: extrafoveal }\end{array}$} \\
\hline 6-7 years & $0.418(0.152)$ & $0.329-0.507$ \\
\hline $8-9$ years & $0.370(0.175)$ & $0.296-0.445$ \\
\hline $10-1 \mid$ years & $0.468(0.169)$ & $0.391-0.544$ \\
\hline Adult & $0.353(0.221)$ & $0.28 I-0.425$ \\
\hline
\end{tabular}

Note: Standard deviations are given in parentheses. $\mathrm{Cl}=$ confidence interval.

that a failure to fixate directly on an approaching vehicle can lead to decrements in looming detection even if the fixation is only a few degrees off target.

Table 2. Effect Sizes for Post Hoc Comparisons of Detection Thresholds Between Age Groups

\begin{tabular}{lcccc}
\hline $\begin{array}{l}\text { Condition and } \\
\text { age group }\end{array}$ & $\begin{array}{c}6-7 \\
\text { years }\end{array}$ & $\begin{array}{c}8-9 \\
\text { years }\end{array}$ & $\begin{array}{c}\text { I0-II } \\
\text { years }\end{array}$ & Adult \\
\hline $\begin{array}{l}\text { Isotropic expansion } \\
\text { 6-7 years }\end{array}$ & - & 0.00 & $0.76^{* *}$ & $1.84^{* * * *}$ \\
8-9 years & 0.00 & - & 0.48 & $1.00^{* * *}$ \\
I0-II years & $1.04^{* *}$ & $0.78^{*}$ & - & $1.09^{* * *}$ \\
Adult & $1.7 I^{* * *}$ & $1.24^{* * *}$ & $0.8 I^{* *}$ & - \\
Expansion with lateral & & & & \\
displacement & & & & \\
6-7 years & - & 0.13 & 0.00 & $0.84^{* *}$ \\
8-9 years & 0.30 & - & 0.12 & $0.73^{*}$ \\
I0-II years & 0.31 & 0.57 & - & $0.80^{* *}$ \\
Adult & 0.36 & 0.10 & 0.61 & - \\
\hline
\end{tabular}

Note: For both isotropic expansion and expansion with displacement, effect sizes (Cohen's $d$ ) for differences in detection thresholds in foveal vision are above the diagonal, and effect sizes (Cohen's $d$ ) for differences in extrafoveal vision are below the diagonal.

$* p<.05 . * * p<.01$. **** $p<.001$.

\section{Detection of expansion with additional lateral displacement}

When the looming vehicle was also displaced laterally, there was a significant effect of age group on detection threshold for looming in foveal vision, $F(3,99)=5.047, p=.003, \eta_{p}{ }^{2}=$ .133 , but not in extrafoveal vision, $F(3,86)=2.142, p=.101$, $\eta_{p}{ }^{2}=.070$ (see Table 1 for summary statistics). Post hoc Tukey HSD analysis of trials in which cars loomed with additional lateral displacement revealed that there were no significant differences between the developmental groups in the threshold for foveal detection, but the adult threshold for foveal detection was significantly better than the threshold for each of the three groups of children (see Table 2 for significance levels and effect sizes). The absence of a difference in detection threshold as a function of age in the extrafoveal condition demonstrates that even adult observers have difficulty detecting looming in the presence of lateral image displacement unless the vehicle is foveated. There were no gender differences in detection threshold for any presentation condition (see Supplementary Documentation and Fig. S1 in the Supplemental Materials for analysis of male and female participants' detection thresholds).

\section{Vehicle-speed thresholds}

Using Equation 4, we converted the observed thresholds into equivalent vehicle speeds for a typical vehicle width $(1.725 \mathrm{~m})$ and a $t_{\mathrm{c}}$ of $5 \mathrm{~s}$.

Detection thresholds for all age groups in the condition in which participants could respond to any discrete edge motion (i.e., foveal trials with isotropic expansion) equated to an ability to detect vehicle approach for speeds in excess of existing urban speed limits (30-40 mph; see Fig. 2a). When edge motion was controlled for (i.e., foveal trials with expansion and displacement), adults could still reliably detect approaching vehicles traveling close to $40 \mathrm{mph}$; children across all age groups, however, were able to reliably detect that a car was moving toward them only if the car was traveling at a speed below approximately $25 \mathrm{mph}$ (see Fig. $2 \mathrm{~b}$ ). Children's perceptual limitations were also evident in their detection of approaching cars in extrafoveal vision, and younger children (6- to 9-year-olds) were unable to reliably detect cars approaching at speeds over $30 \mathrm{mph}$ in either of the extrafoveal conditions (see Figs. 2a and 2b). These threshold speeds are lower than typical urban speed limits in the United Kingdom (30-40 mph), mainland Europe (31-37 $\mathrm{mph}$; Simcic, 2010), and many urban areas in the United States. The threshold speeds are also lower than actual vehicle speeds monitored near the children's schools: We recorded the time taken for randomly selected free-flowing vehicles (vehicles that were not influenced or constrained by a vehicle in front of them) to cover a set distance outside each school $(n=118)$ and calculated an average vehicle speed of $33.92 \mathrm{mph}$ (range: $21-51 \mathrm{mph}$ ). 


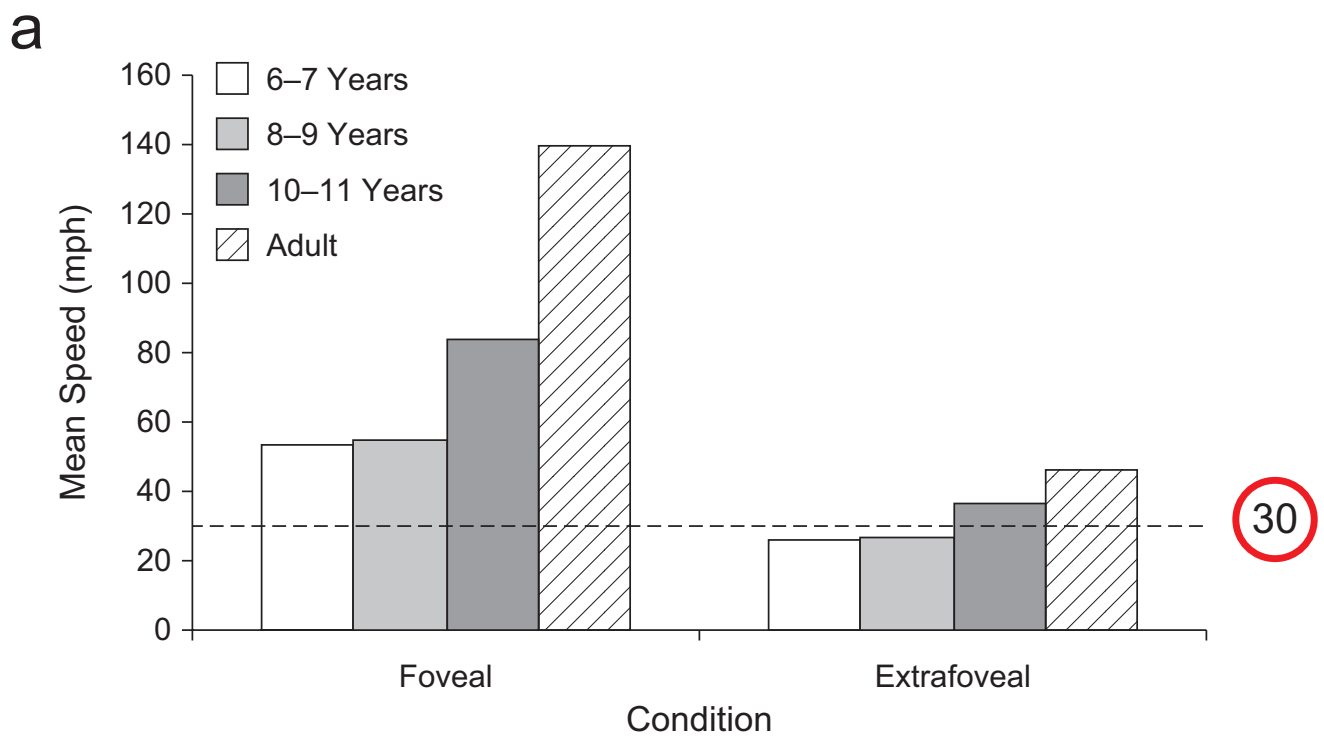

b

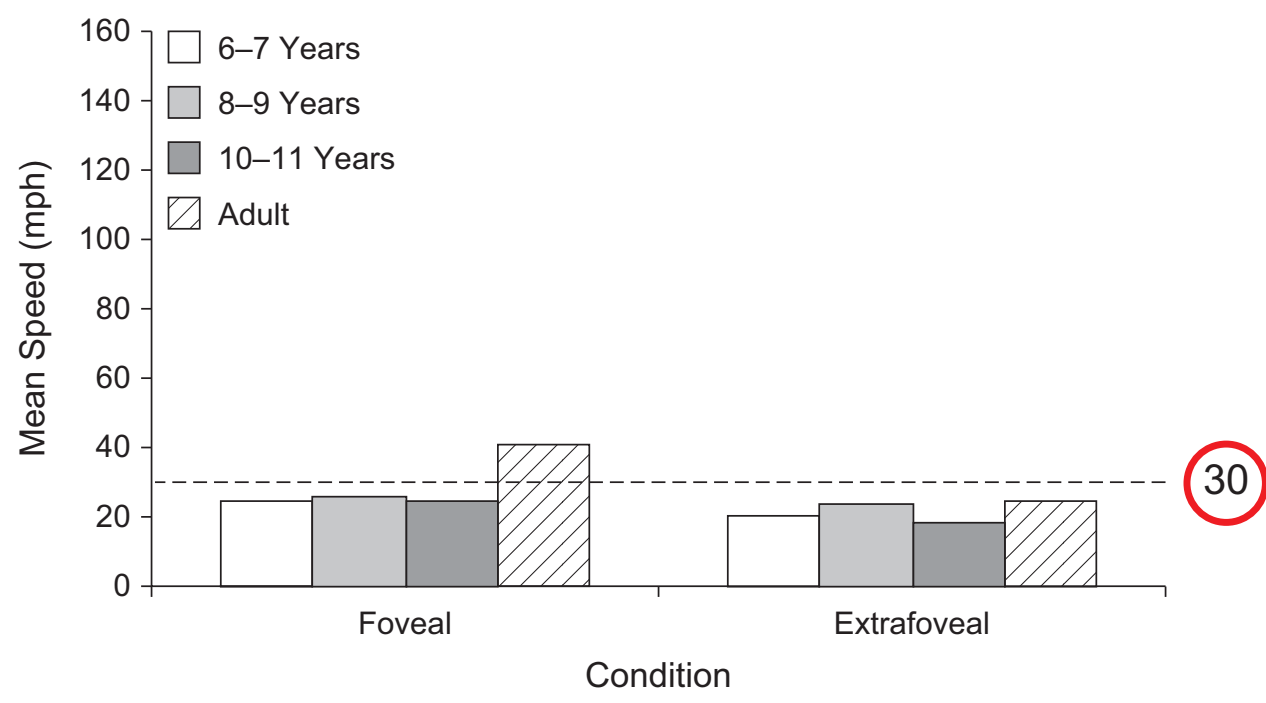

Fig. 2. Mean speed threshold up to which each age group could reliably detect expansion when the object (a) expanded isotropically or (b) expanded isotropically with additional lateral translation. Results are presented separately for objects in foveal and extrafoveal vision. The dotted lines indicate the typical urban speed limit of $30 \mathrm{mph}$.

\section{Discussion}

Our study is the first to demonstrate that the neural mechanisms for detection of looming are not fully developed until adulthood. The perceptual threshold for looming detection has not typically been considered in research on use of tau to estimate TTP. In principle, detection of looming is an essential component in making robust TTP judgments, which dictate effectiveness in skills such as catching, hitting, and road crossing. The developmental trends we observed may explain some of the developmental trends in these activities. We have provided evidence of clear improvements in looming thresholds with increasing age, showing that younger children's poorer perceptual acuity potentially exposes them to greater risk at the roadside.
In this study, we determined that children could not reliably detect a vehicle approaching at speeds higher than approximately $25 \mathrm{mph}$ and did not reach adult levels of perceptual performance under most viewing conditions. The fact that children were able to detect vehicles approaching in excess of $50 \mathrm{mph}$ in the foveal isotropic-expansion condition confirms that this finding was not just an effect of attention. If children are required just to detect any edge motion in central vision, then they can be accurate. But if that motion is even slightly outside of central vision, or if optical expansion occurs in the presence of other scene motion (as might occur during self-motion), then children's performance declines dramatically. The thresholds suggest that when children do not fixate directly on approaching vehicles, or are in motion themselves, they cannot reliably detect the approach 
of vehicles that are $5 \mathrm{~s}$ away and traveling at speeds of $30 \mathrm{mph}$ or higher.

Our findings have important implications for road-safety policy in terms of the upper limits of vehicle speed that allow children to make accurate judgments, and these findings converge with evidence that the risk of pedestrian accidents involving children is nearly 3 times higher in places where mean speeds exceed $25 \mathrm{mph}$ compared with places with lower mean speeds (Roberts, Norton, Jackson, Dunn, \& Hassall, 1995). These data support the case for reduced speed limits outside schools and in other areas densely populated by children (Department of Transport, 1999). Existing research shows that reducing traffic speeds to $20 \mathrm{mph}$ leads to a $50 \%$ reduction in the number of 6- to 11-year-olds who are killed or seriously injured in traffic accidents (Grundy et al., 2009). In part, this reduction is due to speed of impact: Pedestrians have a $90 \%$ chance of surviving being hit by a car traveling under $20 \mathrm{mph}$, but less than a $50 \%$ chance of surviving an impact with a car traveling at $28 \mathrm{mph}$ or higher (Toroyan \& Peden, 2007). However, our results suggest that children's perceptual limitations place them at greater risk of stepping out in front of cars that are traveling at higher speeds. The combined implication is that driving in excess of $20 \mathrm{mph}$ in a residential or school area not only increases the potential severity of any impact with a pedestrian, but also increases the risk that a child will injudiciously cross in front of the vehicle.

\section{Acknowledgments}

We thank the children, parents, and teachers of St. Francis School, St. Michael's School, and James Elliman School, United Kingdom.

\section{Declaration of Conflicting Interests}

The authors declared that they had no conflicts of interest with respect to their authorship or the publication of this article.

\section{Funding}

This work was funded by the United Kingdom Economic \& Social Research Council (Grant ES/F017650/1).

\section{Supplemental Material}

Additional supporting information may be found at http://pss .sagepub.com/content/by/supplemental-data

\section{References}

Cavallo, V., \& Laurent, M. (1988). Visual information and skill level in time-to-collision estimation. Perception, 17, 623-632.

Department for Transport. (1999). Traffic Advisory Leaflet 9/99: 20 mph speed limits and zones. Retrieved January 11, 2011, from
http://www.dft.gov.uk/adobepdf/165240/244921/244924/TAL 9-991.pdf

Department for Transport. (2010). Pedestrian casualties in reported road accidents 2008: Road Accident Statistics Factsheet No. 3. Retrieved March 4, 2010, from http://www.dft.gov.uk/ $\mathrm{pgr} /$ statistics/datatablespublications/accidents/casualtiesgbar/ suppletablesfactsheets/pedestrian2008.pdf

Field, D.T., \& Wann, J.P. (2005). Perceiving time to collision activates the sensorimotor cortex. Current Biology, 15, 1-20.

Grundy, C., Steinbach, R., Edwards, P., Green, J., Armstrong, B., \& Wilkinson, P. (2009). Effect of $20 \mathrm{mph}$ traffic speed zones on road injuries in London, 1986-2006: Controlled interrupted time series analysis. British Medical Journal, 339, b4469. Retrieved December 11, 2009, from http:/www.bmj.com/content/339/bmj.b4469.full

Hoffmann, E.R. (1994). Estimation of time to vehicle arrival - effects of age on use of available information. Perception, 23, 947-955.

Hoffmann, E.R., Payne, A., \& Prescott, S. (1980). Children's estimates of vehicle approach times. Human Factors, 22, 235-240.

Lee, D.N. (1976). A theory of visual control of braking based on information about time-to-collision. Perception, 5, 437-459.

Lieberman, H.R., \& Pentland, A.P. (1982). Microcomputer-based estimation of psychophysical thresholds: The Best PEST. Behavior Research Methods \& Instrumentation, 14, 21-25.

McLeod, R.W., \& Ross, H.E. (1983). Optic-flow and cognitive factors in time-to-collision estimates. Perception, 12, 417-423.

Peron, S., \& Gabbiani, F. (2009). Spike frequency adaptation mediates looming stimulus selectivity in a collision-detecting neuron. Nature Neuroscience, 12, 318-326.

Regan, D., \& Beverley, K.I. (1979). Binocular and monocular stimuli for motion in depth: Changing-disparity and changing-size feed the same motion-in-depth stage. Vision Research, 19, 1331-1342.

Roberts, I., Norton, R., Jackson, R., Dunn, R., \& Hassall, I. (1995). Effects of environmental factors on risk of injury of child pedestrians by motor vehicles: A case-controlled study. British Medical Journal, 310, 91-94.

Schiff, W., \& Detwiler, M.L. (1979). Information used in judging impending collision. Perception, 8, 647-658.

Simcic, G. (2010). Speed Fact Sheet Number 7: Setting appropriate, safe, and credible speed limits. Retrieved November 2, 2010, from http://www.etsc.eu/documents/Speed\%20Fact\%20Sheet\%207.pdf

Sun, H., \& Frost, B.J. (1998). Computation of different optical variables of looming objects in pigeon nucleus rotundus neurons. Nature Neuroscience, 1, 296-303.

Toroyan, T., \& Peden, M. (2007). Youth and road safety. Geneva, Switzerland: World Health Organization.

Tresilian, J.R., Mon-Williams, M., \& Kelly, B.M. (1999). Increasing confidence in vergence as a cue to distance. Proceedings of the Royal Society B: Biological Sciences, 266, 39-44. 